\title{
Real-time molecular-level visualization of mass flow during patterned photopolymerization of liquid-crystalline monomers
}

\author{
Keisuke Ueda', Miho Aizawa ${ }^{2,3}$, Atsushi Shishido (D) ${ }^{2}$ and Martin Vacha (1)
}

\begin{abstract}
Single-particle fluorescence imaging is used to monitor dynamic processes that occur during patterned photopolymerization of liquid-crystalline monomers. A spatial gradient of chemical potential can be created at the border of bright and dark regions by structured illumination in the photopolymerization process, leading to mutual diffusion of polymers and monomers. Analysis of the fluorescence from single quantum dots doped into the monomers at minute concentrations enables visualization of highly directional flow from the illuminated region where the photopolymerization proceeds toward a masked unpolymerized region. This directional mass flow causes flowinduced orientation of the polymers that is subsequently fixed by completion of the polymerization reaction, resulting in a mesoscopic aligned area of the polymer film.
\end{abstract}

\section{Introduction}

The preparation of molecularly aligned structures not only is a fascinating topic from the standpoint of basic molecular physics but also has tremendous technological significance for next-generation optoelectronic, photonic, mechanical, or biomedical materials and devices ${ }^{1-4}$. Traditionally, polymers have been oriented by mechanical processes such as stretching or flow processes such as extrusion. As more advanced methods, photoalignment processes have been developed that enable orientation of side-chain liquid-crystalline (LC) polymers, surfacegrafted polymers, or block copolymers ${ }^{5,6}$. In particular, side-chain LC polymers offer a variety of alignment methods that take advantage of the technology developed for LC molecules by combining with photopolymerization techniques. A disadvantage of methods that involve the

\footnotetext{
Correspondence: Atsushi Shishido (ashishid@res.titech.ac.jp) or

Martin Vacha (vacha.m.aa@m.titech.ac.jp)

${ }^{1}$ Department of Materials Science and Engineering, Tokyo Institute of

Technology, Ookayama 2-12-1-S8-44, Meguro-ku, Tokyo 152-8552, Japan

${ }^{2}$ Laboratory for Chemistry and Life Science, Institute of Innovative Research,

Tokyo Institute of Technology, R1-12, 4259 Nagatsuta, Midori-ku, Yokohama

226-8503, Japan

Full list of author information is available at the end of the article
}

addition of dyes required for photoisomerization, photocrosslinking, or photodecomposition is the inevitable dye residue that degrades the optical properties of the resulting polymer films. On the other hand, methods that allow for alignment without additives, such as the use of photoalignment layers or surface topography templates, have some difficulties in terms of technological complexity and time requirements because these methods involve multistep processes ${ }^{7-10}$.

Recently, a novel and efficient alignment method that does not require the use of additives has been proposed and demonstrated. The method uses spatially patterned unpolarized light to initiate photopolymerization and achieve molecular alignment in an area of a sample where a chemical potential gradient has been created by the presence of polymerized and unpolymerized regions ${ }^{11-14}$. The gradient is assumed to result in mass flow that aligns the precursor monomers and polymers. Continued irradiation further promotes the polymerization and fixes the molecular motion of polymers in the aligned state. The concentration gradient and the following molecular alignment are restricted to a narrow area near the edge of bright and dark regions of the photomask. However, by

\section{(c) The Author(s) 2021}

(c) (i) Open Access This article is licensed under a Creative Commons Attribution 4.0 International License, which permits use, sharing, adaptation, distribution and reproduction cc) in any medium or format, as long as you give appropriate credit to the original author(s) and the source, provide a link to the Creative Commons license, and indicate if changes were made. The images or other third party material in this article are included in the article's Creative Commons license, unless indicated otherwise in a credit line to the material. If material is not included in the article's Creative Commons license and your intended use is not permitted by statutory regulation or exceeds the permitted use, you will need to obtain permission directly from the copyright holder. To view a copy of this license, visit http://creativecommons.org/licenses/by/4.0/. 
scanning such illumination stripes or spots over the sample, it is possible to prepare well-aligned large-scale or patterned LC polymer films ${ }^{12-14}$.

In this work, we aim to provide unambiguous evidence of the phenomenon of mass flow during photopolymerization and its role in the alignment mechanism and to further distinguish between monomer and polymer diffusion. For this purpose, we directly visualize the mass flow occurring near a photomask in real time during the photopolymerization reaction by single-molecule fluorescence imaging ${ }^{15-18}$. The precursor monomer LC film is doped with a very low concentration of highly luminescent quantum dots (QDs), and we use an inverted fluorescence microscope to track the positions of individual QDs. The sample on top of the microscope stage is heated up to $100^{\circ} \mathrm{C}$ by Joule heating using a thin layer of indium thin oxide (ITO) deposited on microscope cover glass ${ }^{19,20}$. The photopolymerization is carried out in situ on the microscope stage by UV laser illumination.

\section{Materials and methods Sample preparation}

The mesogenic monomer 4-[(6-acryloyloxy)hexyloxy]4'-cyanobiphenyl, abbreviated $\mathrm{A} 6 \mathrm{CB}$, was dissolved in spectral grade toluene and mixed with a small amount of dilute toluene solution of $\mathrm{CdSe} / \mathrm{ZnS}$ quantum dots (Lumidot 640, Sigma-Aldrich). The average size of the quantum dots is $6.3 \mathrm{~nm}$ ( $8.6 \mathrm{~nm}$ including ligands), and they are stabilized with hexadecylamine (HDA) and trioctylphosphine (TOPO) ligands. The QDs were chosen for their fluorescence in the red part of the visible spectrum (Fig. S1a), which enabled a significant reduction in the background signal from the bulk of the sample. The mixture was vacuum-oven dried for $2 \mathrm{~h}$, and a small amount of the solid sample was placed on top of a microscope cover glass $(22 \mathrm{~mm} \times 22 \mathrm{~mm} \times 0.15 \mathrm{~mm})$ coated with a thin ITO layer. The ITO had been etched to form a stripe $5 \mathrm{~mm}$ in width with a resistance of $0.5 \mathrm{k} \Omega$. The sample was then heated on a hot plate to above $80^{\circ} \mathrm{C}$, and after melting, it was covered with a smaller piece $(3 \mathrm{~mm} \times 6 \mathrm{~mm})$ of cover glass with a thin layer of $\mathrm{Al}$ as a photomask evaporated on half of its area.

\section{Microscope setup for single-particle tracking and real-time photopolymerization}

Single-particle experiments were performed using an inverted fluorescence microscope (Olympus IX 71) with an oil immersion objective lens (Olympus, UPlan FLN $100 \times / 1.3$ Oil) or an air objective lens (Olympus, PlanFLN $100 \times / 0.95)$. The samples were excited with the $488 \mathrm{~nm}$ line of an Ar ion laser (Coherent Innova 70), and the fluorescence was detected using an electronmultiplying (EM) CCD camera (iXon, Andor Technology) after passing through appropriate optical filters.
The typical acquisition time of the CCD was $100 \mathrm{~ms}$ with 300 gain.

The sample was heated by running a current through the ITO stripe, and its temperature was measured using an infrared thermometer. Typical current-temperature curves for the oil and air objective lenses are shown in Fig. S2. The sample can be heated to $100^{\circ} \mathrm{C}$, and we verified by imaging control samples of polystyrene dispersed QDs that sharp single-particle images can be obtained even at the temperatures used in this experiment $\left(80^{\circ} \mathrm{C}\right)$, as shown in Fig. S1b, c.

Photopolymerization was carried out using a $360 \mathrm{~nm}$ UV laser (UV-FN-360-100mW, CNI), which was expanded five times by a beam expander and reflected by a set of mirrors on top of the microscope stage. The irradiation spot had a diameter of $3 \mathrm{~mm}$, and the typical laser power for the polymerization reaction was $1.5 \mathrm{~mW} \mathrm{~cm}^{-2}$.

Polarization microscopy experiments were performed in transmission mode on the same microscope using a halogen lamp, a pair of polarizers, and additional filters. The whole microscope setup is shown in Fig. S3.

\section{Results and discussion}

The experimental scheme for direct visualization of the photopolymerization is shown in Fig. 1. The monomer (A6CB) doped with red-emitting $\mathrm{CdSe} / \mathrm{ZnS}$ core/shell QDs was prepared by dissolving both components in toluene and mixing them at appropriate concentrations. The mixture was then dried in a vacuum oven, and the powder was deposited on an ITO-covered microscope cover glass and heated on a hot stage above the melting point (to $80^{\circ} \mathrm{C}$ ). After melting, the sample was covered with another microscope cover glass onto which a thin $\mathrm{Al}$ layer had been evaporated as a photomask. This sandwich structure (of an $\sim 5 \mu \mathrm{m}$ thickness) was then cooled down and placed on top of the microscope stage, and the ITO was electrically connected to a power supply. In the photopolymerization experiment, the sample was first heated by the ITO above the melting point and kept in the molten state. The molten state was verified by a transmission microscope image and consequently by monitoring free Brownian diffusion of the QDs in epifluorescence mode. Irradiation of the sample with UV light $\left(360 \mathrm{~nm}, 1.5 \mathrm{~mW} / \mathrm{cm}^{2}\right)$ for several minutes caused the photopolymerization to proceed, and completion of the reaction was confirmed by immobilization of the diffusing QDs, as well as by a change in the transmission image.

To examine the effect of the photomask on molecular alignment during photopolymerization, we carried out real-time monitoring of the reaction under continuous UV irradiation at two different locations, as shown in Fig. 1c. The locations were determined by the $80-\mu \mathrm{m}$ scale of the microscope field of view, and we defined the area in the immediate vicinity of and that partially overlapped 
a)

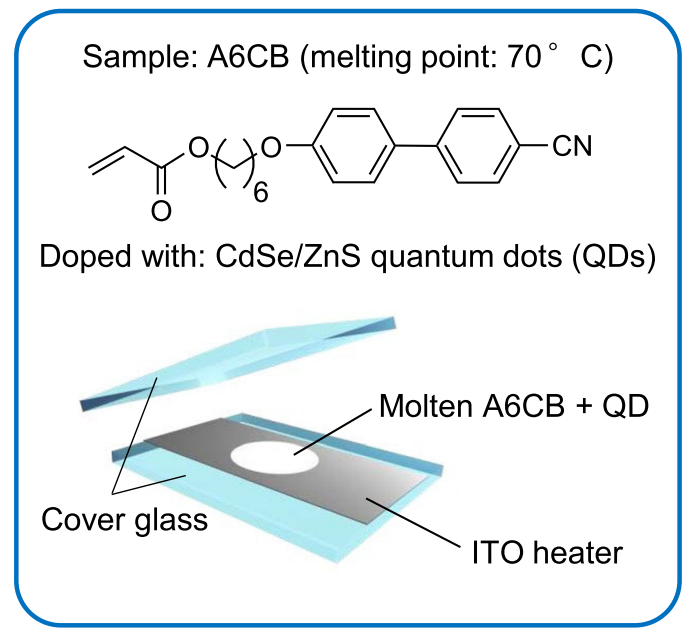

b)

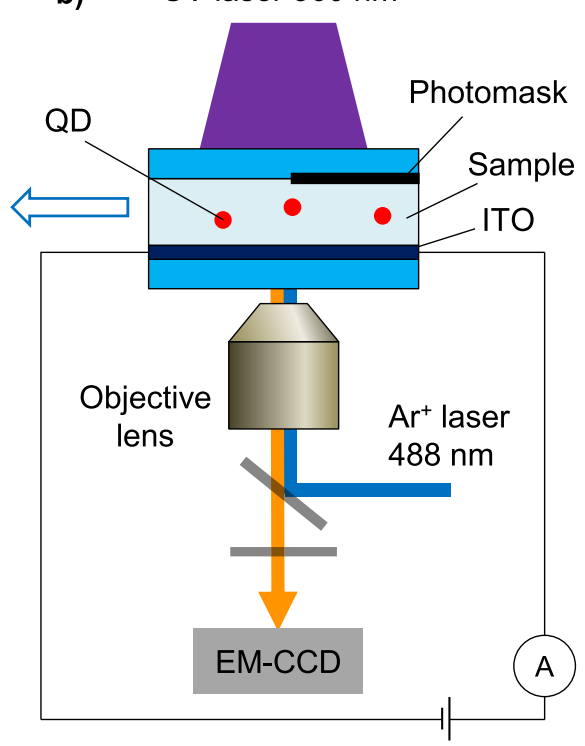

c)

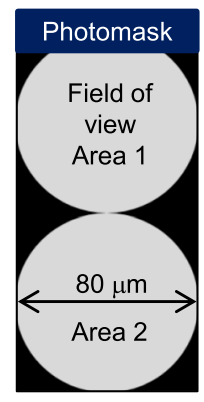

Fig. 1 Experimental scheme for direct real-time visualization of the photopolymerization reaction. a Molecular structure of the $L C$ monomer, and scheme of the sample cell. $\mathbf{b}$ Scheme of the experimental setup. $\mathbf{c}$ Definition of the areas near and far from the photomask where the photopolymerization was monitored.

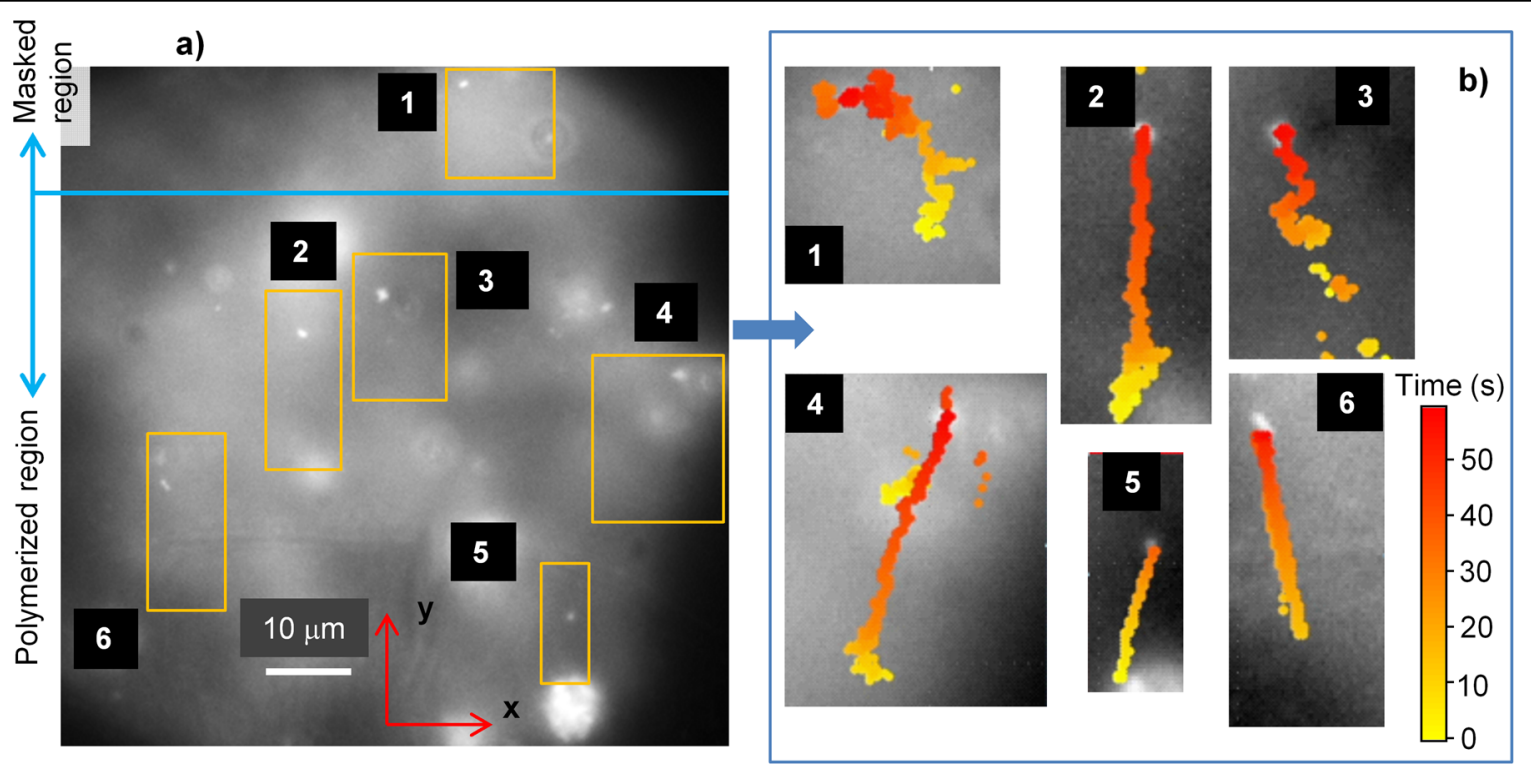

Fig. 2 Fluorescence monitoring of QD diffusion near the photomask. a Fluorescence image of the whole Area 1 near the photomask taken after completion of the photopolymerization reaction. The numbers 1-6 denote individual QDs, and the rectangles are areas magnified in (b); the red arrows define the directions $x$ (parallel to the photomask) and $y$ (perpendicular to it). b Diffusion traces of QDs 1-6 monitored over $60 \mathrm{~s}$ at $0.1 \mathrm{~s}$ steps. The positions in each step are determined from 2D Gaussian fitting of the fluorescence images.

with the photomask as Area 1 and the area further from the photomask as Area 2. The main observations in Area 1 (close to the photomask) are summarized in Fig. 2. The overall fluorescence image of Area 1 after completion of the photopolymerization is shown in Fig. 2a, with a blue horizontal line indicating the edge of the mask. The numbers 1-6 denote examples of individual QDs that were tracked during the photopolymerization. The individual traces of the diffusing QDs are shown in Fig. 2b. More examples are shown in Fig. S4. The UV irradiation 
was switched on $120 \mathrm{~s}$ before the start of the traces, i.e., at the value of ' 0 ' on the time scale in Fig. $2 b$, the irradiation had been on for $2 \mathrm{~min}$. The traces were constructed from 600 consecutive fluorescence images taken every $0.1 \mathrm{~s}$ over the time span of $1 \mathrm{~min}$. In each image, the position of the particular QD was determined as the centroid of a 2D Gaussian fit of the fluorescence spot.

QD 1 is located in the masked region where photopolymerization does not proceed during the experiment. This is reflected in the random Brownian motion of QD 1 throughout the measured time interval. To visualize the random nature of the diffusion, we analyzed the diffusion lengths of the QDs within individual intervals of $0.1 \mathrm{~s}$. The lengths were analyzed separately in the directions parallel to the photomask edge (direction $x$ ) and perpendicular to it (direction $y$ ), as indicated in Fig. 2a. The results (plotted in Fig. 3a) confirm that the extent and direction of the diffusion in the masked region (in the molten state of the monomer) are completely random without any visible tendency.

Compared to the above results, QDs 2-6, which are located in the region where photoirradiation does occur during the measurement time, behave in a completely different way (Fig. 2b). In the early stages of the UV irradiation, they show random Brownian diffusion, similar to QD 1. As the polymerization reaction partially proceeds, the diffusion considerably slows down and becomes highly anisotropic. This trend is visualized by the plot of the diffusion lengths in the $x$ and $y$ directions of QD 2 in Fig. 3b. The scatter of the points during the first $30 \mathrm{~s}$ of the experiment resembles that of QD 1 (Fig. 3a). At 40-60 s, the magnitude of the diffusion lengths decreases, and the lengths in the $y$ direction (perpendicular to the mask, blue dots) are significantly larger than those in the $x$ direction (parallel to the mask, red dots). This reflects the directionality of the diffusion toward the photomask edge. Therefore, it is reasonable to assume that the polymerization had proceeded, causing directional diffusion of QD 2 in this time range.

Interestingly, the observations in Fig. 2 also indicate that during the measurement window, the polymerization itself proceeds gradually, starting far from the photomask (at the bottom of the image) and approaching the photomask at the top of the image as time proceeds. This is reflected by the different patterns of the diffusion traces of the QDs. Those at the bottom (far from the photomask), such as QD 5 and QD 6, show mainly directional diffusion because at the start of the measurement window, they are in the region where polymerization has proceeded to some extent. In contrast, the traces closer to the photomask, such as QD 2 and QD 3, initially show random Brownian diffusion, which changes to directional flow as the polymerization proceeds further up (from the bottom of the image). More such examples are shown in Fig. S4, where the trend is visible for QD A, QD B, and QD C in that order.

To quantify these observations, we further calculated diffusion coefficients $D_{x}$ and $D_{y}$ in the respective directions $x$ and $y$ using mean square displacement $(M S D)$ analy$\mathrm{sis}^{21,22}$. The $M S D$ values $\left\langle x_{i}^{2}(t / \tau)\right\rangle$ were typically constructed over the time $t$ of $5 \mathrm{~s}$ from 50 data points of the diffusion lengths with intervals $\tau=0.1 \mathrm{~s}$. The diffusion coefficients were determined from the MSD slope $\left\langle x_{i}^{2}(t / \tau)\right\rangle=2 D t$. The diffusion coefficients calculated for statistical ensembles of the data are shown in the histograms in Fig. S5. Analysis of the random Brownian diffusion at different time points in the early stages of photopolymerization (such as $0-30 \mathrm{~s}$ in Fig. $3 \mathrm{~b}$ ) for 20 individual QDs provides a large distribution of the diffusion coefficients with an average $D_{x}$ of $0.025 \mu^{2} \mathrm{~s}^{-1}$ and an average $D_{y}$ of $0.027 \mu^{2} \mathrm{~s}^{-1}$ (Fig. S5a). Figure 3c shows the distribution of the ratio $D_{y} / D_{x}$ for individual data points during the $0-30 \mathrm{~s}$ interval. The average of this ratio of 1.2 confirms the random nature of the diffusion. Analysis of the anisotropic directional diffusion of the 20 QDs observed in the later stages (such as 40-60 s in Fig. $3 \mathrm{~b}$ ) indicates an order of magnitude smaller average $D_{x}$ of $0.0020 \mu \mathrm{m}^{2} \mathrm{~s}^{-1}$ and widely distributed $D_{y}$ values with an average of $0.0054 \mu \mathrm{m}^{2} \mathrm{~s}^{-1}$ (Fig. S5b). The distribution of the ratio $D_{y} / D_{x}$ during the $40-60 \mathrm{~s}$ interval shown in Fig. $3 \mathrm{~d}$ has an average value of 3.7 , confirming the directional nature of the diffusion toward the photomask edge.

To further verify the effect of the interface on the diffusion behavior of the QDs, we examined the photopolymerization process in Area 2 (as defined in Fig. 1c), the center of which is $\sim 100 \mu \mathrm{m}$ from the photomask edge. Figure S6a shows an example of the diffusion traces of three QDs in a randomly selected section of Area 2. The range of diffusion is apparently smaller than that in Area 1 (Fig. 2). The diffusion of one of these QDs was visualized by plotting the diffusion length per $0.1 \mathrm{~s}$ in Fig. S6b. At $<10 \mathrm{~s}$ in Fig. S6b, this QD shows random Brownian diffusion, similar to QDs $\mathbf{1}$ and $\mathbf{2}$ in Fig. 3. In the later stage of photopolymerization, however, the diffusion completely stops in both the $x$ and $y$ directions, as reflected by the 0 values of the diffusion lengths. The same results were also obtained on control samples that were prepared without the use of a photomask (Fig. S7). In such samples, photopolymerization proceeded homogeneously throughout the sample, and anisotropic directional diffusion of QDs was not observed.

Based on the above observations, we have confirmed that the patterned photopolymerization causes diffusion of the QDs in a direction perpendicular to the boundary of the photomask and that this effect is observable within $<70 \mu \mathrm{m}$ from the mask edge. Further, we need to verify that in a sample prepared under the same conditions, the resulting LC polymer is actually aligned in one direction 

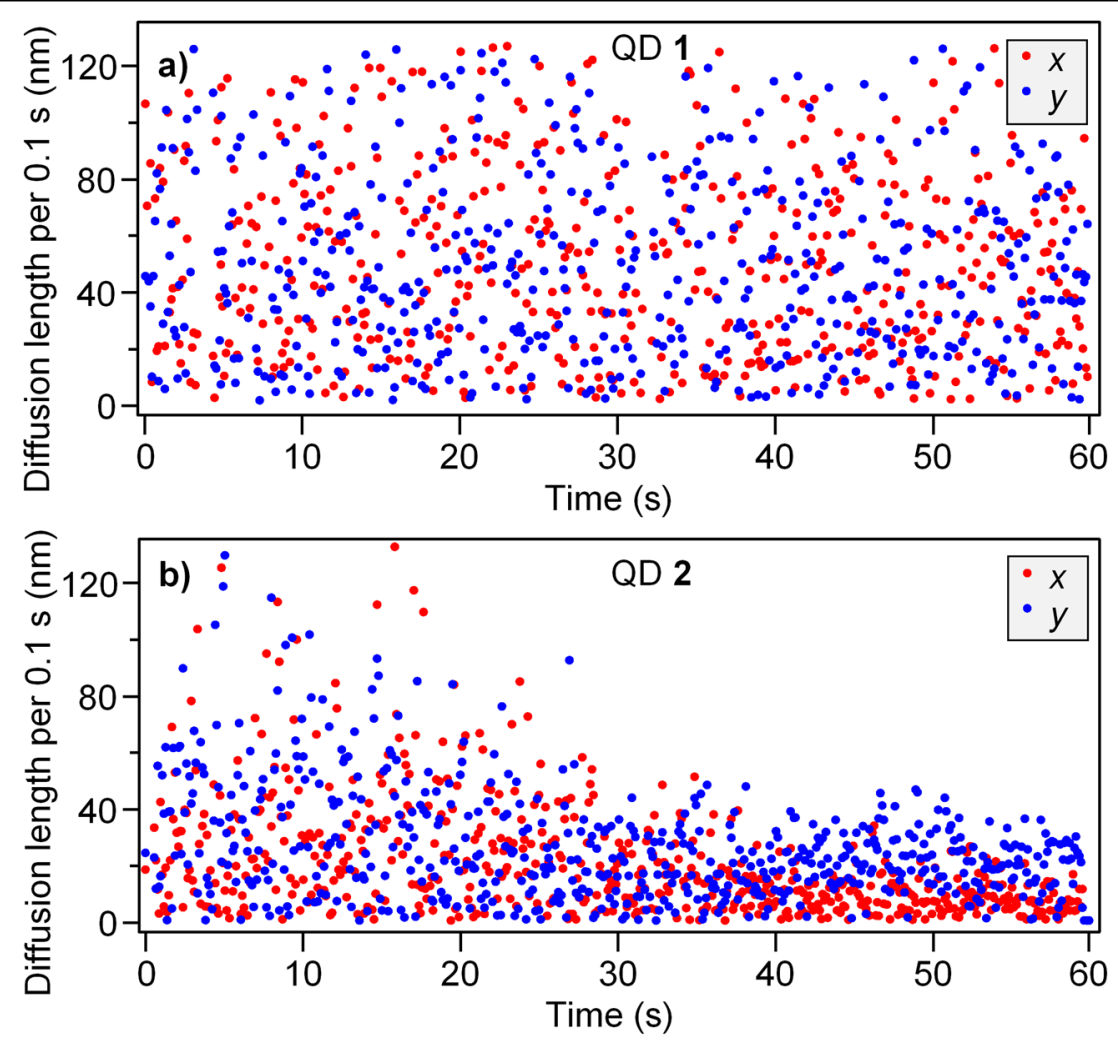

Early stage (0-30 s)

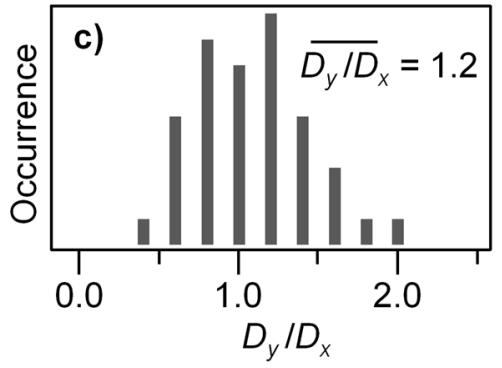

Later stage (40-60 s)

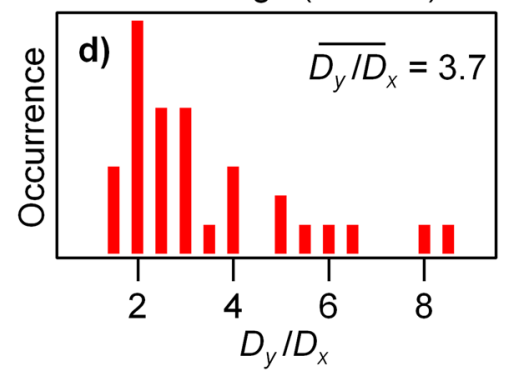

Fig. 3 Analysis of QD diffusion near the photomask. a Diffusion length per 0.1 s of QD 1 analyzed separately along the $x$ (red symbols) and $y$ (blue symbols) directions during the observation time of $60 \mathrm{~s}$. UV irradiation started 2 min before the displayed interval and continued throughout the measurement. b The same as (a) for QD 2. c Distribution of the ratio $D_{x} / D_{y}$ obtained by analysis of 20 QDs and calculated from the diffusion coefficients $D_{x}$ and $D_{y}$ within the interval 0-30 s. d Same as (c) analyzed within the interval 40-60 s.

in a narrow mesoscopic region near the photomask. For this purpose, we imaged such a sample using a polarized optical microscope, as shown in Fig. S8. The image clearly shows that LCs are aligned only in the illuminated region near the edge of the photomask.

A mechanism proposed earlier to account for the molecular alignment during photopolymerization assumed the formation of a chemical potential gradient between the unpolymerized masked region and the partially polymerized irradiated region at the interface ${ }^{12}$. Under this concentration gradient, both monomers and polymers diffuse perpendicular to the mask edge in opposite directions. The anisotropic diffusion creates a thermodynamic force that induces macroscopic mass flow in the direction perpendicular to the interface. This mass flow causes orientation of the polymer main chains and mesogenic side chains by shear stress, and the subsequent polymerization immobilizes their orientation. We believe that the directional diffusion of QDs from the partially polymerized region toward the photomask observed in this work directly reflects this proposed mass flow ${ }^{12}$. Considering the chemical potential gradient, a mass flow from the polymerized region is directed into the area of unpolymerized monomers. The process of molecular alignment through mass flow is schematically shown in Fig. 4. Before photopolymerization, the QDs undergo free Brownian diffusion 
a) Before polymerization

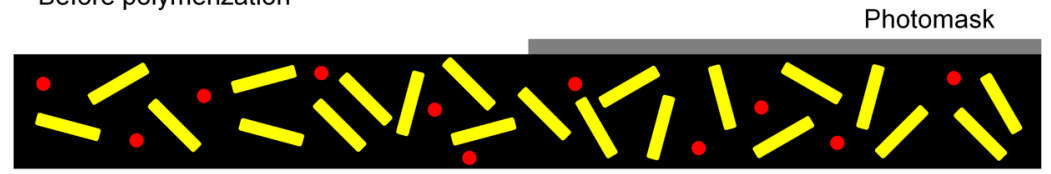

b)

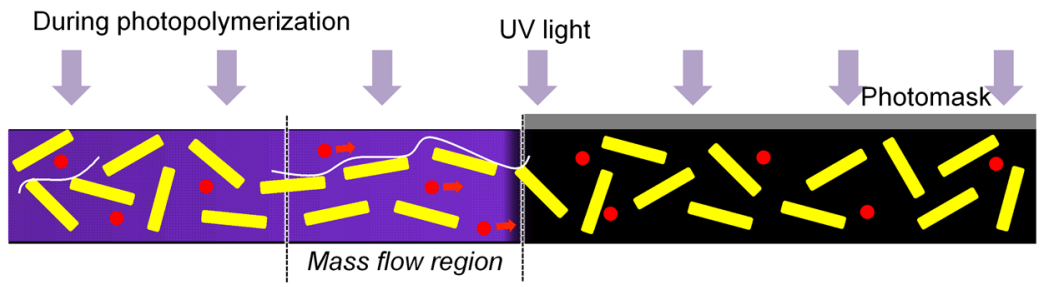

c)

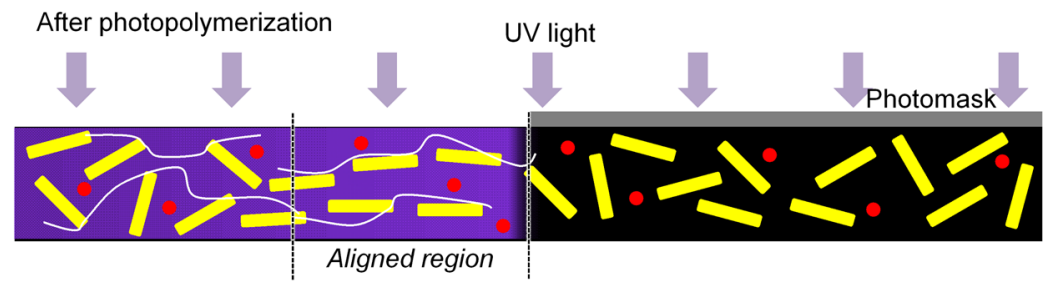

Fig. 4 Schematic illustration of the mechanism of molecular alignment during photopolymerization near a photomask. a Before photopolymerization; b during photopolymerization; c after photopolymerization. Yellow rods, red dots, and white lines indicate mesogenic sidechain units, quantum dots, and polymer main chains, respectively.

in the molten isotropic state, with relatively large diffusion constants (Fig. 4a). Upon UV irradiation, polymers are formed at a low concentration in the irradiated region (Fig. 4b). At the interface between the irradiated and unirradiated regions, the concentration gradient of the polymers forms a chemical potential, and the polymers diffuse into the masked region, producing mass flow. The QDs acting as a visualizer of the mass flow also diffuse directionally toward the mask edge. In the region distant from the photomask, the polymers effectively immobilize the QDs, as seen in Fig. S6b. Finally, continued irradiation completes the polymerization and fixes the alignment of the polymer main chain and mesogenic side chains perpendicular to the photomask (Fig. 4c). In principle, there should be mutual diffusion of the polymers and monomers in opposite directions. The directed motion of QDs toward the unirradiated region observed in this work indicates that the mass flow is dominated by polymer diffusion. Other mechanisms accounting for the motion of QDs might be possible. Lee et al. showed that QDs can reflect the mass flow of an unpolymerized LC in the nematic phase as well ${ }^{22}$, and Maeda et al. reported that competing mechanisms of diffusiophoresis and thermophoresis coexist in threecomponent systems ${ }^{23,24}$. However, the directed motion of QDs observed in this work must be induced by diffusion of polymers, and the unidirectional diffusion of polymer chains resulted in molecular alignment during the patterned photopolymerization.

\section{Conclusions}

In conclusion, we used single-particle fluorescence imaging to reveal the molecular-level physical processes underlying the alignment of LCs during photopolymerization with structured light. We unambiguously showed that the photopolymerization is accompanied by macroscopic mass flow of side-chain LC polymers, which results in flow-induced orientation that is subsequently fixed by completion of the polymerization reaction. These results will not only have an impact in the field of polymer physics, where they will contribute to the knowledge on molecular alignment, but also be valuable for the further development of film orientation technology.

\section{Acknowledgements}

The research was financially supported by JSPS KAKENHI grant number 19H02684 and by JSPS KAKENHI Grant Number JP26107014 in Scientific Research on Innovative Areas "Photosynergetics" (M.V.). Further, this work was supported by JSPS KAKENHI grant number JP17H05250 in Scientific Research on Innovative Areas "Photosynergetics", by Grant-in Aid for Scientific Research on Innovative Areas "Molecular Engine" (JSPS KAKENHI grant number JP18H05422), and by JST CREST grant number JPMJCR1814, Japan (A.S.). This work was performed under the Research Program of "Dynamic Alliance for Open Innovation Bridging Human, Environment and Materials" in "Network Joint Research Center for Materials and Devices".

\section{Author details}

'Department of Materials Science and Engineering, Tokyo Institute of Technology, Ookayama 2-12-1-S8-44, Meguro-ku, Tokyo 152-8552, Japan. 'Laboratory for Chemistry and Life Science, Institute of Innovative Research, Tokyo Institute of Technology, R1-12, 4259 Nagatsuta, Midori-ku, Yokohama 226-8503, Japan. ${ }^{3}$ Present address: Research Institute for Sustainable Chemistry, 
National Institute of Advanced Industrial Science and Technology, 1-1-1 Higashi, Tsukuba, Ibaraki 305-8565, Japan

\section{Conflict of interest}

The authors declare no competing interests.

\section{Publisher's note}

Springer Nature remains neutral with regard to jurisdictional claims in published maps and institutional affiliations.

Supplementary information The online version contains supplementary material available at https://doi.org/10.1038/s41427-021-00292-1.

Received: 25 November 2020 Revised: 24 January 2021 Accepted: 29 January 2021.

Published online: 19 March 2021

\section{References}

1. White, T. J. \& Broer, D. J. Programmable and adaptive mechanics with liquid crystal polymer networks and elastomers. Nat. Mater. 14, 1087-1098 (2015).

2. Hirst, A. R., Escuder, B., Miravet, J. F. \& Smith, D. K. High-tech applications of selfassembling supra-molecular nanostructured gel-phase materials: from regenerative medicine to electronics devices. Angew. Chem. Int. Ed. 47, 8002-8018 (2008)

3. Woltman, S. J., Jay, G. D. \& Crawford, G. P. Liquid-crystal materials find a new order in biomedical applications. Nat. Mater. 6, 929-938 (2007).

4. O'Neill, M. \& Kelly, S. M. Liquid crystals for charge transport, luminescence, and photonics. Adv. Mater. 15, 1135-1146 (2003).

5. Yaroshchuk, O. \& Reznikov, Y. Photoalignment of liquid crystals: basics and current trends. J. Mater. Chem. 22, 286-300 (2012).

6. Seki, T., Nagano, S. \& Hara, M. Versatility of photoalignment techniques: from nematics to a wide range of functional materials. Polymer 54, 6053-6072 (2013).

7. Seki, T. A wide array of photoinduced motions in molecular and macromolecular assemblies at interfaces. Bull. Chem. Soc. Jpn. 91, 1026-1057 (2018).

8. Gupta, V. K. \& Abbott, N. L. Design of surfaces for patterned alignment of liquid crystals on planar and curved substrates. Science 276, 1533-1536 (1997).
9. Xia, Y., Cedillo-Servin, G., Kamien, R. D. \& Yang, S. Guided folding of nematic liquid crystal elastomer sheets into 3D via patterned 1D microchannels. Adv. Mater. 28, 9637-9643 (2016)

10. Lin, R. \& Rogers, J. A. Molecular-scale soft imprint lithography for alignment layers in liquid crystal devices. Nano Lett. 7, 1613-1621 (2007).

11. Hisano, K. et al. Alignment layer-free molecular ordering induced by masked photopolymerization with non-polarized light. Appl. Phys. Express 9, 072601 (2016).

12. Hisano, K. et al. Scanning wave photopolymerization enables dye-free alignment patterning of liquid crystals. Sci. Adv. 3, e1701610 (2017).

13. Hisano, K. et al. Single-step creation of polarization gratings by scanning wave photopolymerization with unpolarized light. J. Opt. Soc. Am. B 36, D112-D118 (2019).

14. Aizawa, M. et al. Direct fabrication of a q-plate array by scanning wave photopolymerization. J. Opt. Soc. Am. B 36, D47-D51 (2019).

15. Ito, S. et al. Evaluation of diffusion coefficient in a dextrin-based photo-curable material by single molecule tracking. Appl. Phys. Express 2, 075004 (2009).

16. Woell, D. et al. Polymers and single molecule fluorescence spectroscopy, what can we learn? Chem. Soc. Rev. 38, 313-328 (2009).

17. Vacha, M. \& Habuchi, S. Conformation and physics of polymer chains: a singlemolecule perspective. NPG Asia Mater. 2, 134-142 (2010).

18. Woell, D. et al. Radical polymerization tracked by single molecule spectroscopy. Angew. Chem. Int. Ed. 47, 783-787 (2008).

19. Suzuki, K., Habuchi, S. \& Vacha, M. Blinking of single dye molecules in a polymer matrix is correlated with free volume in polymers. Chem. Phys. Lett. 505, 157-160 (2011).

20. Flier, B. M. I. et al. Heterogeneous diffusion in thin polymer films as observed by high-temperature single-molecule fluorescence microscopy. J. Am. Chem. Soc. 134, 480-488 (2012).

21. Habuchi, S., Sato, N., Yamamoto, T., Tezuka, Y. \& Vacha, M. Multimode diffusion of ring polymer molecules revealed by a single-molecule study. Angew. Chem. Int. Ed. 49, 1418-1421 (2010)

22. Lee, S., Noda, K. Hirata, S. \& Vacha, M. Position-dependent three-dimensional diffusion in nematic liquid crystal monitored by single-particle fluorescence localization and tracking. J. Phys. Chem. Lett. 6, 1403-1407 (2015).

23. Maeda, Y. T., Buguin, A. \& Libchaber, A. Thermal separation: interplay between the soret effect and entropic force gradient. Phys. Rev. Lett. 107, 038301 (2011).

24. Jiang, H. R., Wada, H., Yoshinaga, N. \& Sano, M. Manipulation of colloids by a nonequilibrium depletion force in a temperature gradient. Phys. Rev. Lett. 102 208301 (2009). 